Supporting Information

Metal Atoms Collinear with the Spiro Carbon of 6,6-Open Adducts, $\mathrm{M}_{2} @ \mathrm{C}_{80}(\mathrm{Ad})$

( $\mathrm{M}=\mathrm{La}$ and $\mathrm{Ce}, \mathrm{Ad}=$ adamantylidene $)$

Michio Yamada, ${ }^{\dagger}$ Chika Someya, ${ }^{\dagger}$ Takatsugu Wakahara, ${ }^{\dagger}$ Takahiro Tsuchiya, ${ }^{\dagger}$ Yutaka Maeda, ${ }^{\ddagger}$ Takeshi Akasaka, ${ }^{* \dagger}{ }^{\dagger}$ Kenji Yoza,${ }^{\S}$ Ernst Horn, ${ }^{\perp}$ Michael T. H. Liu, ${ }^{\circ}$ Naomi Mizorogi," and Shigeru Nagase*,\#

Contribution from the Center for Tsukuba Advanced Research Alliance, University of Tsukuba, Tsukuba, Ibaraki 305-8577, Japan, Department of Chemistry, Tokyo Gakugei University, Koganei, Tokyo 184-8501, Japan, Bruker AXS, K. K., Yokohama, Kanagawa 221-0022, Japan, Department of Chemistry, Rikkyo University, Tokyo 171-8501, Japan,

University of Prince Edward Island, Charlottetown, Prince Edward Island, Canada

C1A4P3, and Department of Theoretical and Computational Molecular Science, Institute for Molecular Science, Okazaki, Aichi 444-8585, Japan

E Mail: akasaka@tara.tsukuba.ac.jp

Table of Contents

Complete refs 12 and 15 S2

$\begin{array}{ll}\text { Figure S1. HPLC profiles of the reaction mixtures. } & \text { S3 }\end{array}$

Figure S2. HPLC profile and MALDI-TOF mass spectrum of $2 . \quad$ S4

Figure S3. MALDI-TOF mass spectrum of ${ }^{13} \mathrm{C}$-enriched $\mathrm{Ce}_{2} @ \mathrm{C}_{80} . \quad$ S4

Figure S4. HPLC profile and MALDI-TOF mass spectrum of ${ }^{13} \mathrm{C}$-enriched $3 . \quad$ S4

Figure S5. Cyclic and differential pulse voltammogram of 2 . S5

Figure S6. Cyclic and differential pulse voltammogram of $\mathbf{3}$ S5

Figure S7. HMQC NMR spectrum of $2 . \quad$ S6

$\begin{array}{ll}\text { Figure S8. }{ }^{13} \mathrm{C} \text { NMR spectrum of } 2 . & \text { S7 }\end{array}$

$\begin{array}{ll}\text { Figure S9. Line-fitting plot; chemical shift vs. } T^{-1} \text { of } 3 . & \text { S7 }\end{array}$

Figure S10. Line-fitting plot; chemical shift vs. $T^{-2}$ of $3 . \quad$ S8

Figure S11. The electrostatic potential map of $\left[\mathrm{C}_{80}(\mathrm{Ad})\right]^{6-}$ S8

Table S1. Summary of Crystallographic Data of 2 at 103, 133, 188, and 273 K S9

Table S2. Total Energies (Hartree) and Cartesian Coordinates ( $\AA$ ) of $\mathrm{La}_{2} @ \mathrm{C}_{80}(\mathrm{Ad}) \mathrm{S} 10$ 
Complete ref. 12

Frisch, M. J.; Trucks, G. W.; Schlegel, H. B.; Scuseria, G. E.; Robb, M. A.; Cheeseman, J. R.; Montgomery, Jr., J. A.; Vreven, T.; Kudin, K. N.; Burant, J. C.; Millam, J. M.; Iyengar, S. S.; Tomasi, J.; Barone, V.; Mennucci, B.; Cossi, M.; Scalmani, G.; Rega, N.; Petersson, G. A.; Nakatsuji, H.; Hada, M.; Ehara, M.; Toyota, K.; Fukuda, R.; Hasegawa, J.; Ishida, M.; Nakajima, T.; Honda, Y.; Kitao, O.; Nakai, H.; Klene, M.; Li, X.; Knox, J. E.; Hratchian, H. P.; Cross, J. B.; Adamo, C.; Jaramillo, J.; Gomperts, R.; Stratmann, R. E.; Yazyev, O.; Austin, A. J.; Cammi, R.; Pomelli, C.; Ochterski, J. W.; Ayala, P. Y.; Morokuma, K.; Voth, G. A.; Salvador, P.; Dannenberg, J. J.; Zakrzewski, V. G.; Dapprich, S.; Daniels, A. D.; Strain, M. C.; Farkas, O.; Malick, D. K.; Rabuck, A. D.; Raghavachari, K.; Foresman, J. B.; Ortiz, J. V.; Cui, Q.; Baboul, A. G.; Clifford, S.; Cioslowski, J.; Stefanov, B. B.; Liu, G.; Liashenko, A.; Piskorz, P.; Komaromi, I.; Martin, R. L.; Fox, D. J.; Keith, T.; Al-Laham, M. A.; Peng, C. Y.; Nanayakkara, A.; Challacombe, M.; Gill, P. M. W.; Johnson, B.; Chen, W.; Wong, M. W.; Gonzalez, C.; Pople, J. A. GAUSSIAN 03, Revision C. 01, Gaussian Inc., Wallingford, CT, 2004.

Complete ref. 15

Maeda, Y.; Matsunaga, Y.; Wakahara, T.; Takahashi, S.; Tsuchiya, T.; Ishitsuka, M. O.; Hasegawa, T.; Akasaka, T.; Liu, M. T. H.; Kokura, K.; Horn, E.; Yoza, K.; Kato, T.; Okubo, S.; Kobayashi, K.; Nagase, S.; Yamamoto, K. J. Am. Chem. Soc. 2004, 126, 6858-6859. 

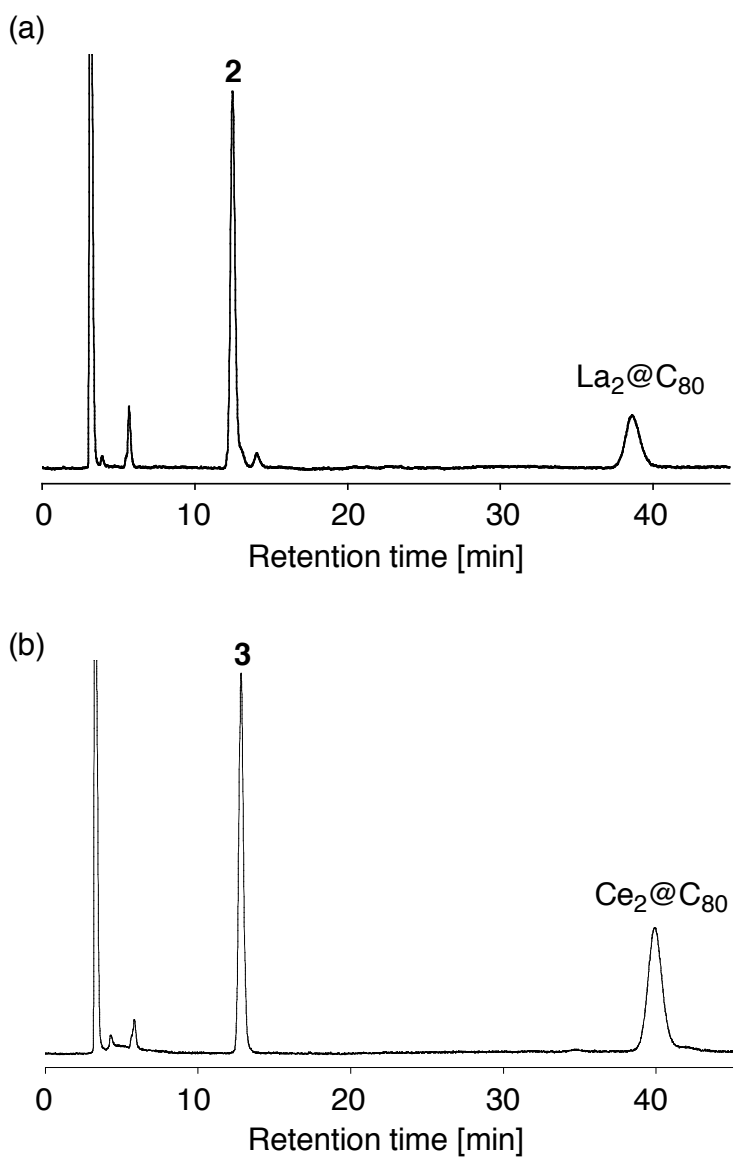

Figure S1. HPLC profiles of the reaction mixtures for photochemical reactions of (a) $\mathrm{La}_{2} @ \mathrm{C}_{80}$ with 1 and (b) $\mathrm{Ce}_{2} @ \mathrm{C}_{80}$ with 1. Conditions: column, Buckyprep (4.6 mm $\times$ $250 \mathrm{~mm}$ i.d.); eluent, toluene $1.0 \mathrm{~mL} / \mathrm{min}$. 

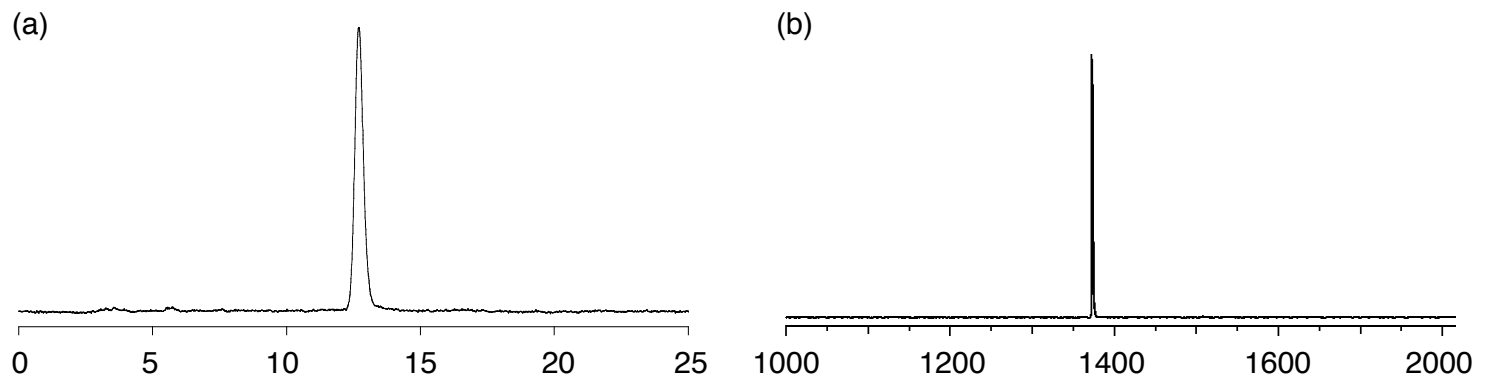

Figure S2. (a) HPLC profiles of 2. Conditions: column, Buckyprep (4.6 mm $\times 250 \mathrm{~mm}$ i.d.); eluent, toluene $1.0 \mathrm{~mL} / \mathrm{min}$. (b) MALDI-TOF mass spectrum of 2.
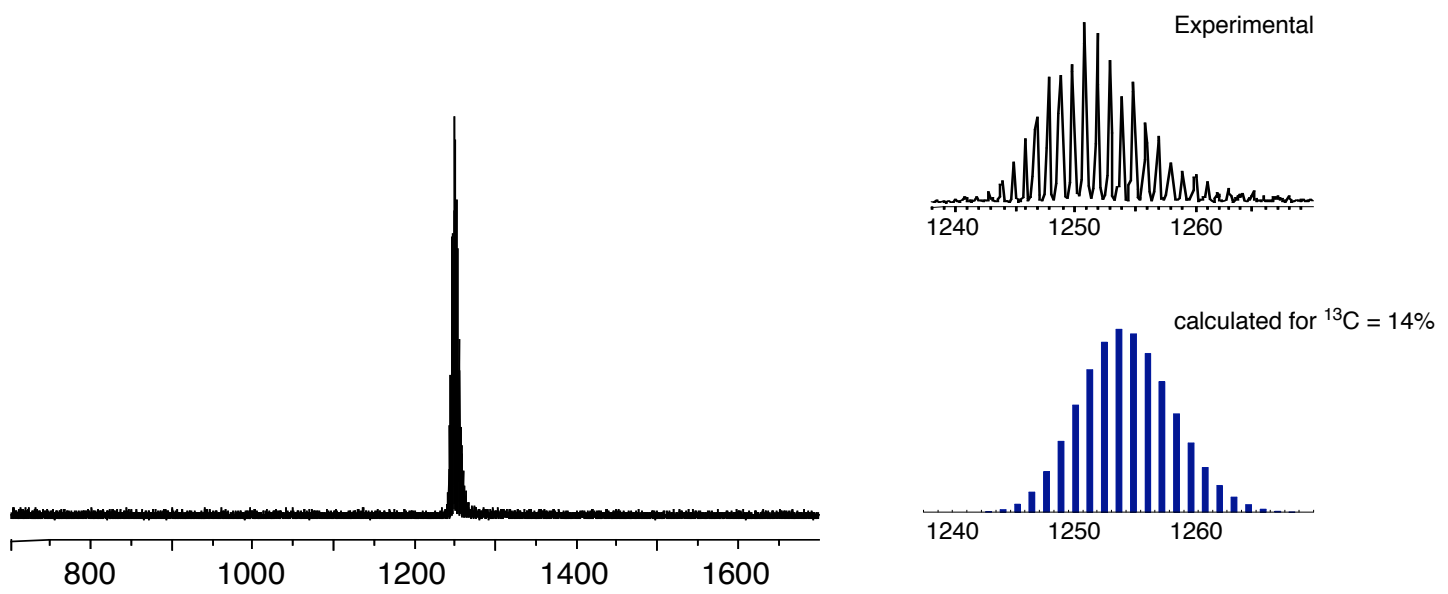

Figure S3. MALDI-TOF mass spectrum of ${ }^{13} \mathrm{C}$-enriched $\mathrm{Ce}_{2} @ \mathrm{C}_{80}$.

(a)

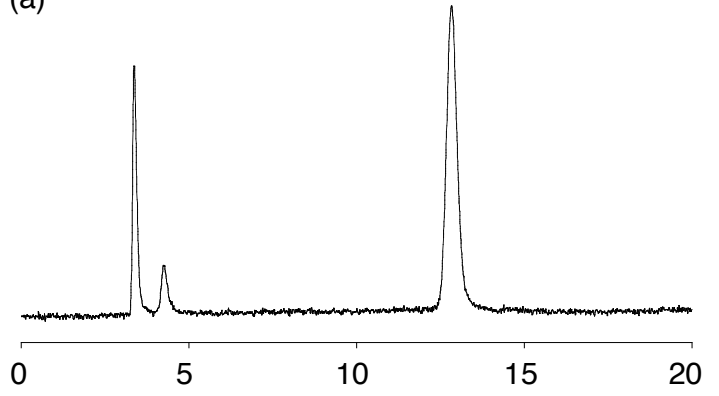

(b)

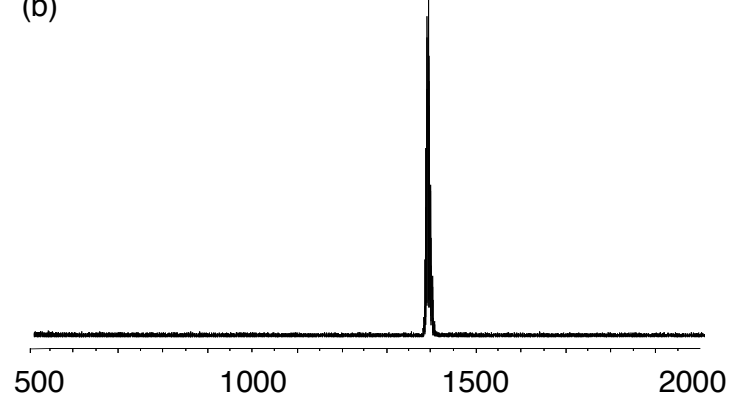

Figure S4. (a) HPLC profiles of ${ }^{13} \mathrm{C}$-eniched 3. Conditions: column, Buckyprep (4.6 mm $\times 250 \mathrm{~mm}$ i.d.); eluent, toluene $1.0 \mathrm{~mL} / \mathrm{min}$. (b) MALDI-TOF mass spectrum of ${ }^{13} \mathrm{C}$ enriched 2. 


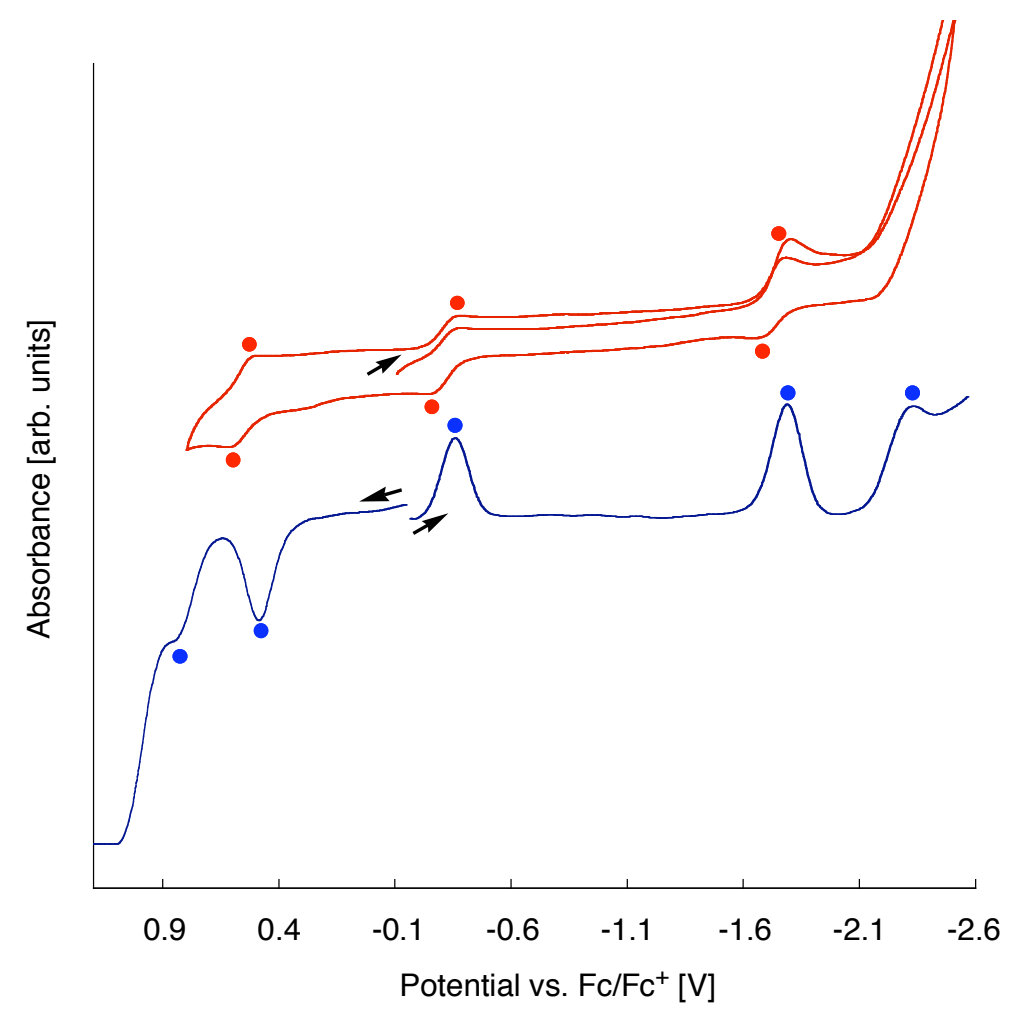

Figure S5. Cyclic and differential pulse voltammogram of 2.

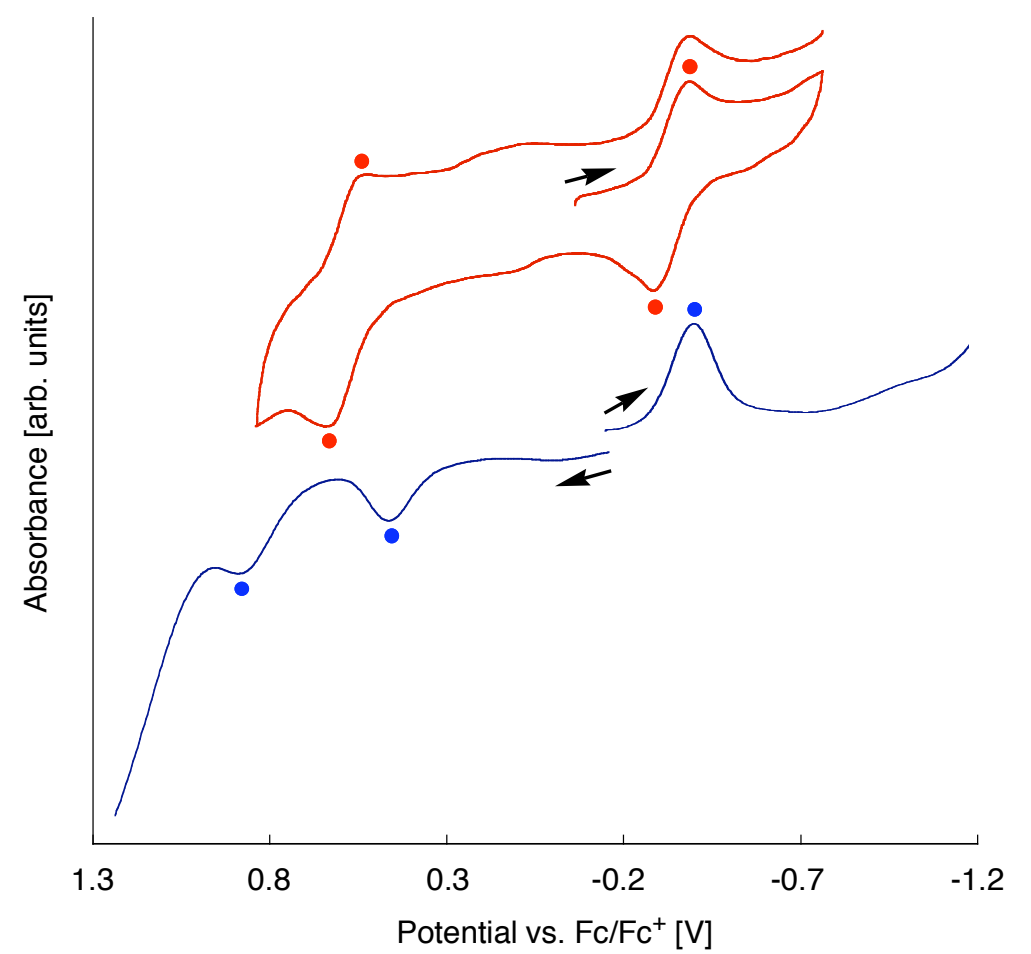

Figure S6. Cyclic and differential pulse voltammogram of $\mathbf{3}$. 

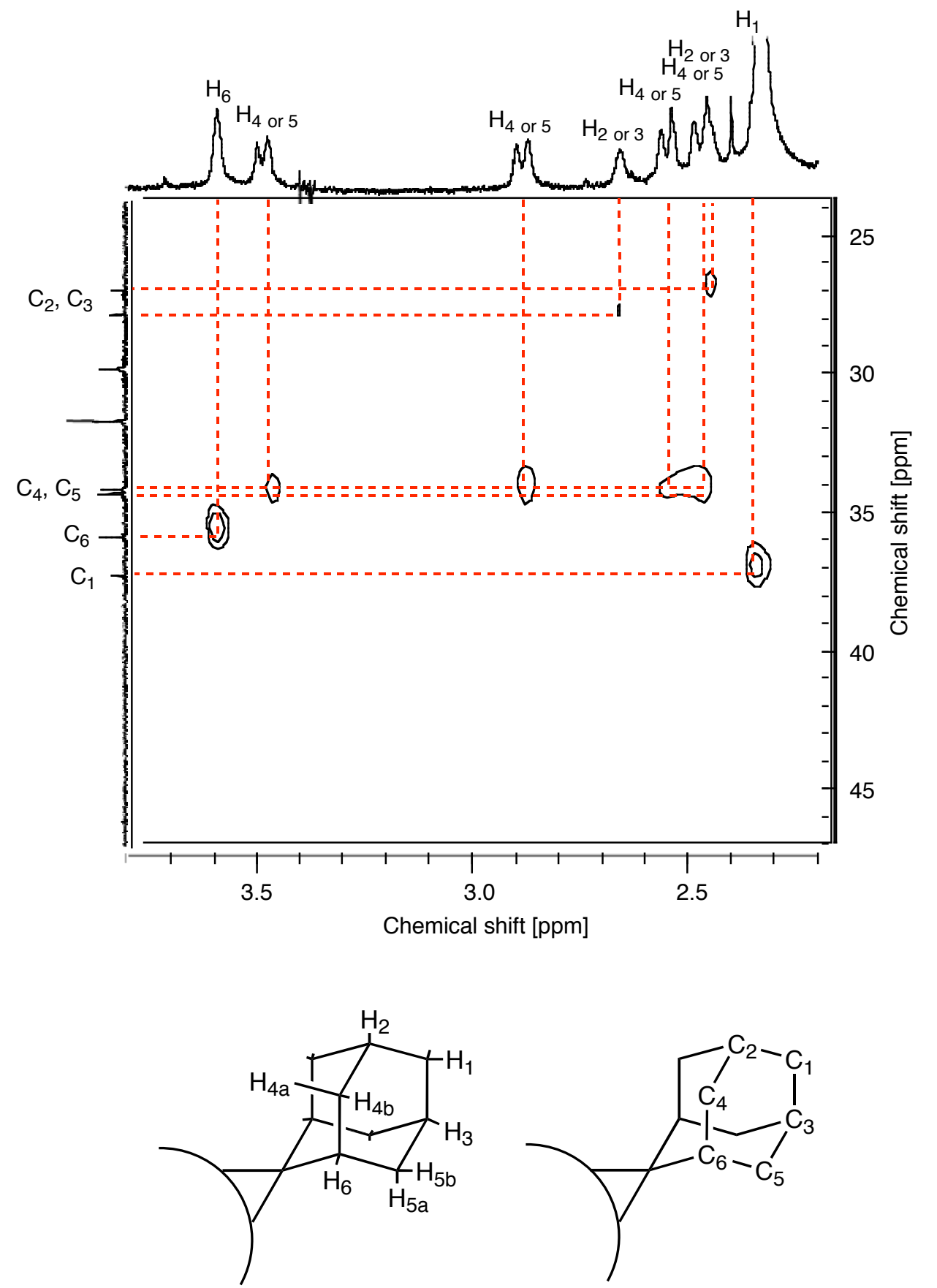

Figure S7. HMQC NMR spectrum of 2 in $\mathrm{C}_{2} \mathrm{D}_{2} \mathrm{Cl}_{4} / \mathrm{CS}_{2}$ at $303 \mathrm{~K}$. 


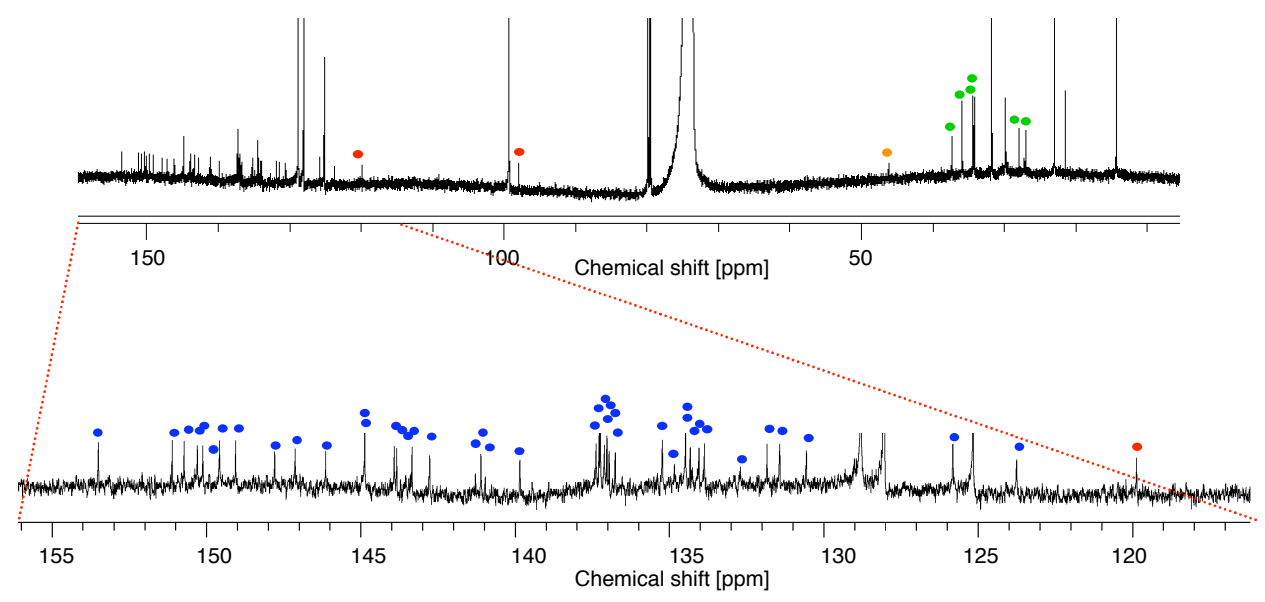

Figure S8. ${ }^{13} \mathrm{C}$ NMR spectrum of 2 in $\mathrm{C}_{2} \mathrm{D}_{2} \mathrm{Cl}_{4} / \mathrm{CS}_{2}$ at $303 \mathrm{~K}$. The signals marked by blue, red, orange and green solid circles are due to the $\mathrm{sp}^{2}$-carbon atoms on the cage, $\mathrm{sp}^{2}$-carbon atoms on the addition position of the cage, spiro carbon on the Ad group, and the other carbon atoms on the Ad group, respectively.

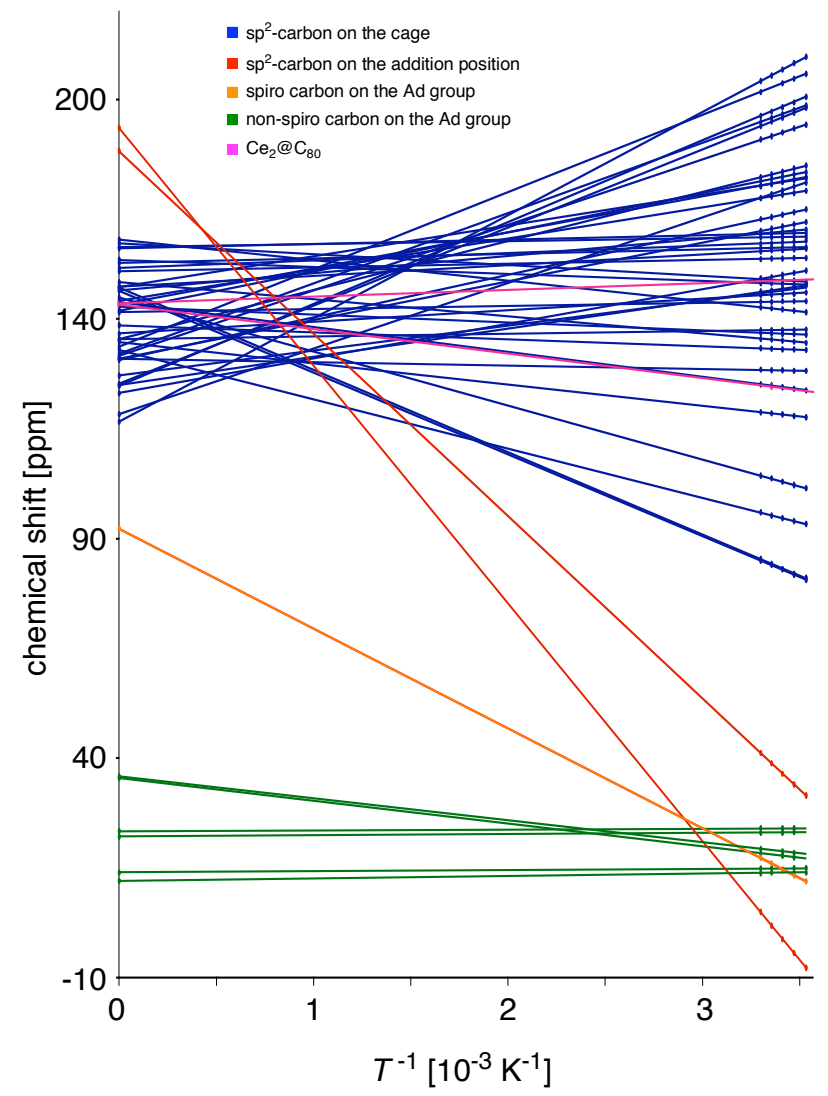

Figure S9. Line-fitting plot; chemical shift vs. $T^{-1}$ of $\mathbf{3}$. 


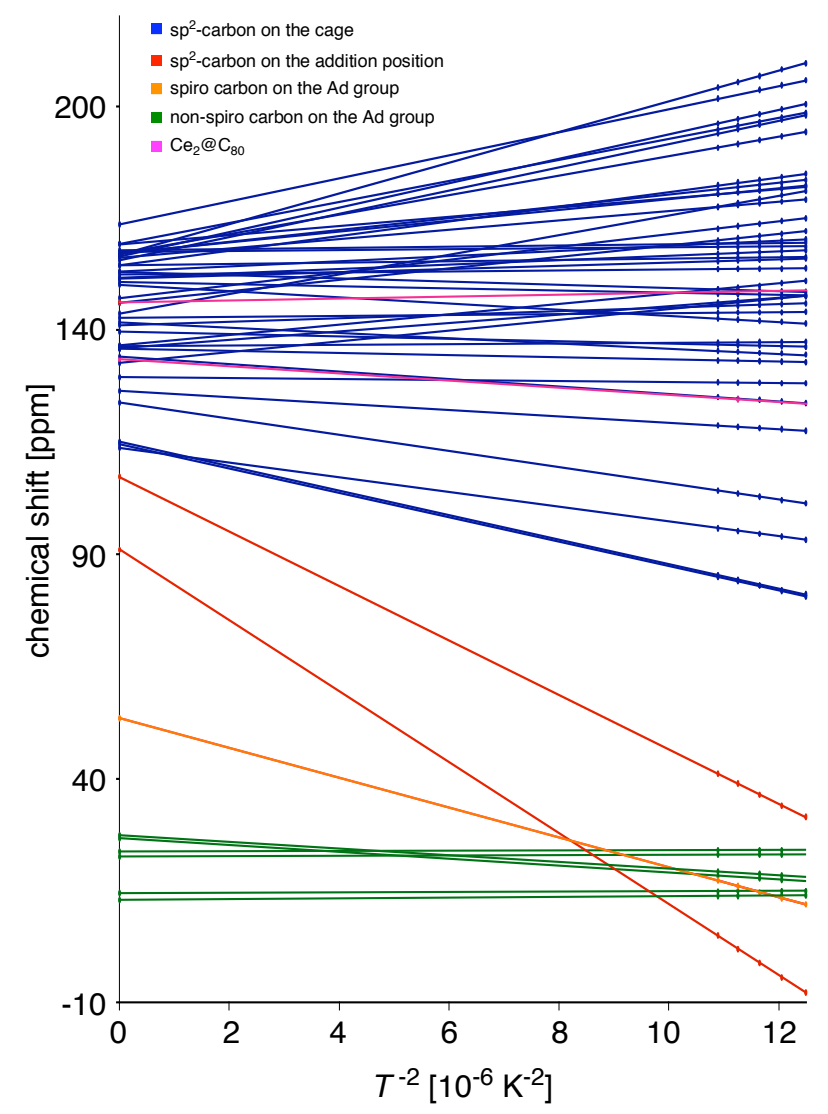

Figure S10. Line-fitting plot; chemical shift vs. $T^{-2}$ of 3 .

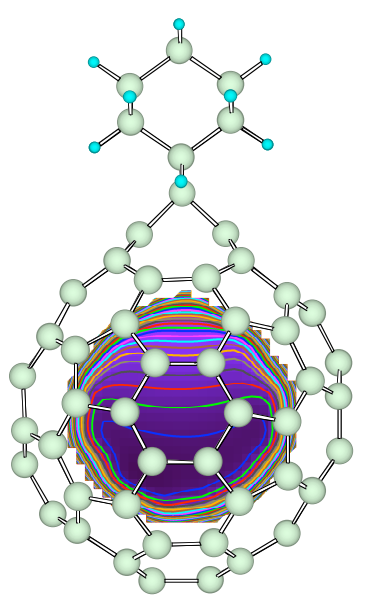

Figure S11. The electrostatic potential map of $\left[\mathrm{C}_{80}(\mathrm{Ad})\right]^{6-}$. 
Table S1. Summary of Crystallographic Data of 2 at 103, 133, 188, and 273 K

\begin{tabular}{|c|c|c|c|c|}
\hline Temperature & $103 \mathrm{~K}$ & $133 \mathrm{~K}$ & $188 \mathrm{~K}$ & $273 \mathrm{~K}$ \\
\hline Formula & $\mathrm{C}_{96} \mathrm{H}_{18} \mathrm{Cl}_{2} \mathrm{La}_{2}$ & $\mathrm{C}_{96} \mathrm{H}_{18} \mathrm{Cl}_{2} \mathrm{La}_{2}$ & $\mathrm{C}_{96} \mathrm{H}_{18} \mathrm{Cl}_{2} \mathrm{La}_{2}$ & $\mathrm{C}_{96} \mathrm{H}_{18} \mathrm{Cl}_{2} \mathrm{La}_{2}$ \\
\hline formula weight & 1519.82 & 1519.82 & 1519.82 & 1519.82 \\
\hline wavelength, $\AA$ & 0.71075 & 0.71075 & 0.71075 & 0.71075 \\
\hline crystal system & Orthorhombic & Orthorhombic & Orthorhombic & Orthorhombic \\
\hline space group & Pbca & Pbca & Pbca & Pbca \\
\hline$a, \AA$ & 21.944(3) & $21.958(3)$ & $21.966(3)$ & $22.031(3)$ \\
\hline$b, \AA$ & $22.017(2)$ & $22.035(2)$ & $22.066(3)$ & $22.122(3)$ \\
\hline$c, \AA$ & $20.452(2)$ & $20.4625(17)$ & $20.473(2)$ & $20.498(2)$ \\
\hline$\alpha, \operatorname{deg}$ & 90.00 & 90.00 & 90.00 & 90.00 \\
\hline$\beta, \operatorname{deg}$ & 90.00 & 90.00 & 90.00 & 90.00 \\
\hline$\gamma, \operatorname{deg}$ & 90.00 & 90.00 & 90.00 & 90.00 \\
\hline Volume, $\AA^{3}$ & $9881(2)$ & $9900.8(17)$ & $9924(2)$ & $9990(2)$ \\
\hline$Z$ & 8 & 8 & 8 & 8 \\
\hline$D_{\text {calc }}, \mathrm{Mg} / \mathrm{m}^{3}$ & 2.043 & 2.039 & 2.035 & 2.021 \\
\hline Absorption coefficient & 1.884 & 1.880 & 1.876 & 1.864 \\
\hline$F(000)$ & 5936 & 5936 & 5936 & 5936 \\
\hline$\theta$ range, deg & 3.09 to 27.49 & 3.09 to 27.42 & 3.09 to 27.48 & 3.08 to 27.48 \\
\hline \multirow[t]{3}{*}{ Limiting indices } & $-28<=h<=28$ & $-28<=h<=28$ & $-28<=h<=28$ & $-27<=h<=28$ \\
\hline & $-23<=k<=28$ & $-24<=k<=28$ & $-23<=k<=28$ & $-24<=k<=28$ \\
\hline & $-25<=l<=26$ & $-26<=l<=26$ & $-25<=l<=26$ & $-26<=l<=26$ \\
\hline Reflections collected & 77189 & 89327 & 77851 & 67464 \\
\hline Independent reflections & 11216 & 11244 & 11266 & 11273 \\
\hline data / restraints / parameters & 11216 / 834 / 909 & 11244 / 834 / 909 & 11266 / 834 / 909 & 11273 / 834 / 901 \\
\hline$R_{\text {int }}$ & 0.0784 & 0.0796 & 0.0783 & 0.0858 \\
\hline$R_{1}[I>2 \sigma(I)]$ & 0.0602 & 0.0590 & 0.0593 & 0.0746 \\
\hline$w R_{2}[I>2 \sigma(I)]$ & 0.1631 & 0.1609 & 0.1582 & 0.1800 \\
\hline$R_{1}$ [all data] & 0.0806 & 0.0757 & 0.0843 & 0.1178 \\
\hline$w R_{2}$ [all data] & 0.1714 & 0.1680 & 0.1675 & 0.1945 \\
\hline GOF on $F^{2}$ & 1.218 & 1.232 & 1.148 & 1.192 \\
\hline max, min peaks, e/ $\AA^{3}$ & $3.904,-1.304$ & $3.918,-1.220$ & $2.086,-1.407$ & $3.559,-0.894$ \\
\hline
\end{tabular}


Table S2 Total Energies (Hartree) and Cartesian Coordinates $(\AA)$ of $\mathrm{La}_{2} @ \mathrm{C}_{80}(\mathrm{Ad})$

Structure a

Total energy $=-3500.43798213$

La -0.0052101 .2206270 .000000$

La $0.018793-2.6847430 .000000$

C 3.2926651 .0721031 .446665

C 2.6609310 .7354522 .670578

C $2.646974-0.6189503 .133972$

C $1.414880-0.8240913 .849761$

C $0.739216-2.0789493 .804767$

C 3.9776430 .0594280 .720363

C $3.943807-1.3015041 .167690$

C $3.239084-1.6607242 .351998$

C $2.597752-2.9449762 .365747$

C $1.368080-3.1508943 .080716$

C $3.926406-2.1511830 .000000$

C $3.227866-3.3878080 .000000$

C $2.605509-3.8104001 .223350$

C -3.281798-2.807758 1.460741

C -2.673941 -2.442874 2.696998

C 1.4330271 .3934263 .085301

C - $1.450978-3.0752593 .141676$

C -2.658251-1.081357 3.142229

C 0.6731440 .4056013 .816168

C -0.682782 -2.079659 3.856003

C - $1.423789-0.8511523 .851011$

C -0.7438670 .4031253 .766742$

C 2.7147472 .1582620 .717711

C -2.660236 -3.8758430.718338

C -3.968145 -1.8016960.722621

C 1.6078372 .9587671 .189190

C - $1.483491-4.5700031 .178055$ 
C -3.950538 -0.436709 1.167574

C 0.8343242 .4747622 .352777

C $-0.825590-4.1083652 .388454$

C -3.245113-0.058166 2.345366

C - 0.6368862 .5311662 .362995

C $0.611755-4.1620422 .400025$

C -2.601758 1.2250102 .343747

C - 1.3648471 .4685673 .030188

C 1.0599313 .6211570 .000000

C -0.734212 -5.0414540.000000

C -3.9511500.4120080.000000

C - 1.1113543 .6439760 .000000

C $0.744917-5.0526370 .000000$

C -3.2867921.6631770.000000

C - 1.5117872 .9745011 .228218

C $1.379800-4.5994691 .228511$

C -2.655742 2.0890741 .216275

C -0.010034 4.6865540.000000

C 0.0030245 .6084621 .258910

C 1.2720846 .4879201 .250278

C 1.2827447 .3874640 .000000

C - 1.2407747 .4127030 .000000

C - 1.2479906 .5139051 .251183

C 0.0298678 .2880890 .000000

H -0.003097 5.0011592 .166715

H 1.2844017 .0991362 .162304

H 2.1737695 .8623881 .277479

H 2.1873138 .0088630 .000000

H -2.132573 8.0523200 .000000

H -2.1624015.906753 1.279317

H -1.248194 7.125987 2.162633

H 0.0362668 .9429800 .882201

C $3.2926651 .072103-1.446665$ 
C $2.6609310 .735452-2.670578$

C $2.646974-0.618950-3.133972$

C $1.414880-0.824091-3.849761$

C $0.739216-2.078949-3.804767$

C $3.9776430 .059428-0.720363$

C $3.943807-1.301504-1.167690$

C $3.239084-1.660724-2.351998$

C $2.597752-2.944976-2.365747$

C 1.368080 -3.150894-3.080716

C $2.605509-3.810400-1.223350$

C -3.281798-2.807758-1.460741

C -2.673941-2.442874-2.696998

C $1.4330271 .393426-3.085301$

C - $1.450978-3.075259-3.141676$

C -2.658251-1.081357-3.142229

C $0.6731440 .405601-3.816168$

C -0.682782 -2.079659-3.856003

C - $1.423789-0.851152-3.851011$

C -0.743867 0.403125 -3.766742

C $2.7147472 .158262-0.717711$

C - 2.660236-3.875843-0.718338

C -3.968145 -1.801696-0.722621

C $1.6078372 .958767-1.189190$

C - $1.483491-4.570003-1.178055$

C -3.950538 -0.436709-1.167574

C $0.8343242 .474762-2.352777$

C -0.825590 -4.108365-2.388454

C -3.245113-0.058166-2.345366

C -0.636886 2.531166-2.362995

C $0.611755-4.162042-2.400025$

C -2.601758 $1.225010-2.343747$

C - $1.3648471 .468567-3.030188$

C - $1.5117872 .974501-1.228218$ 
C $1.379800-4.599469-1.228511$

C -2.655742 2.089074-1.216275

C $0.0030245 .608462-1.258910$

C $1.2720846 .487920-1.250278$

C -1.247990 6.513905 -1.251183

H -0.003097 $5.001159-2.166715$

H $1.2844017 .099136-2.162304$

H $2.1737695 .862388-1.277479$

H -2.162401 $5.906753-1.279317$

H -1.248194 7.125987 -2.162633

H $0.0362668 .942980-0.882201$

\section{Structure b}

Total energy $=-3500.40264465$

La -0.003530 -0.795828 1.846157

La $-0.003530-0.795828-1.846157$

C 3.2748321 .0669981 .431793

C 2.6227600 .7116452 .659147

C $2.624568-0.6385323 .143287$

C $1.439425-0.8341763 .955313$

C $0.765488-2.1308623 .937068$

C 3.9570720 .0468290 .716808

C $3.931684-1.3205441 .167118$

C $3.235161-1.6868522 .353999$

C $2.603058-2.9624102 .387848$

C $1.364975-3.1554273 .123296$

C $3.910911-2.1656420 .000000$

C $3.195244-3.3976570 .000000$

C $2.579714-3.8055161 .227702$

C -3.281867-2.765007 1.445455

C -2.640657 -2.385461 2.682241

C 1.4328171 .3808083 .100558 
C - $1.449825-3.0336573 .149883$

C - $2.637299-1.0297563 .147916$

C 0.6866110 .4506223 .938740

C - $0.691945-2.0903153 .956698$

C - $1.445298-0.8091883 .953557$

C -0.7714510 .4893083 .922120$

C 2.7147952 .1495720 .715889

C - $2.705832-3.8438810 .719448$

C - $3.957703-1.7457390 .719358$

C 1.5774842 .9085231 .174947

C - $1.497333-4.4881441 .166904$

C -3.941564-0.374840 1.166246

C 0.8399222 .4541752 .310809

C - $0.833546-4.0685292 .355289$

C -3.2409430 .0035292 .346853$

C -0.6048972 .5193782 .340161$

C $0.589086-4.1382442 .387308$

C - 2.5973391 .2744682 .364515

C - 1.3570931 .4966463 .079811

C 1.0222443 .5662450 .000000

C $-0.750024-4.8816360 .000000$

C -3.9324050 .4697510 .000000$

C -0.9525133 .5505280 .000000$

C $0.675114-4.8637540 .000000$

C -3.2286501 .7061560 .000000$

C - 1.4104662 .9205441 .220396

C $1.334205-4.5289211 .226929$

C - 2.5976592 .1127501 .214698

C 0.0332114 .6884400 .000000

C 0.0306625 .5968011 .259039

C 1.2866076 .4951141 .250936

C 1.2823717 .3943640 .000000

C - 1.2427647 .3786160 .000000 
C - 1.2354976 .4805941 .252318

C 0.0143768 .2741940 .000000

H 0.0349314 .9863842 .164837

H 1.2932467 .1079022 .162311

H 2.194352 5.8787001 .274809

H 2.1760468 .0319140 .000000

H -2.144148 8.0052090 .000000

H -2.136378 5.8540211 .278314

H -1.248789 7.094892 2.162558

H 0.0103048 .9291000 .882440

C $3.2748321 .066998-1.431793$

C $2.6227600 .711645-2.659147$

C $2.624568-0.638532-3.143287$

C $1.439425-0.834176-3.955313$

C $0.765488-2.130862-3.937068$

C $3.9570720 .046829-0.716808$

C $3.931684-1.320544-1.167118$

C $3.235161-1.686852-2.353999$

C $2.603058-2.962410-2.387848$

C 1.364975 -3.155427 -3.123296

C $2.579714-3.805516-1.227702$

C -3.281867-2.765007-1.445455

C -2.640657 -2.385461 -2.682241

C $1.4328171 .380808-3.100558$

C - $1.449825-3.033657-3.149883$

C -2.637299-1.029756 -3.147916

C $0.6866110 .450622-3.938740$

C -0.691945 -2.090315 -3.956698

C - $1.445298-0.809188-3.953557$

C $-0.7714510 .489308-3.922120$

C $2.7147952 .149572-0.715889$

C - $2.705832-3.843881-0.719448$

C -3.957703-1.745739-0.719358 
C $1.5774842 .908523-1.174947$

C -1.497333 -4.488144 - 1.166904

C -3.941564 -0.374840-1.166246

C $0.8399222 .454175-2.310809$

C -0.833546 -4.068529-2.355289

C -3.240943 $0.003529-2.346853$

C -0.604897 2.519378 -2.340161

C $0.589086-4.138244-2.387308$

C -2.597339 $1.274468-2.364515$

C -1.357093 1.496646-3.079811

C -1.410466 2.920544-1.220396

C $1.334205-4.528921-1.226929$

C -2.597659 2.112750-1.214698

C $0.0306625 .596801-1.259039$

C $1.2866076 .495114-1.250936$

C - $1.2354976 .480594-1.252318$

H $0.0349314 .986384-2.164837$

H $1.2932467 .107902-2.162311$

H $2.1943525 .878700-1.274809$

H -2.136378 $5.854021-1.278314$

H - $1.2487897 .094892-2.162558$

H $0.0103048 .929100-0.882440$

Structure c

Total energy $=-3500.29013654$

$\begin{array}{rccc}\mathrm{La} & 1.7750000 & -2.7500000 & 0.0000000 \\ \mathrm{La} & -1.8580000 & -2.7500000 & 0.0000000 \\ \mathrm{C} & 3.2270000 & -0.9530000 & 1.4190000 \\ \mathrm{C} & 2.5980000 & -1.3110000 & 2.6470000 \\ \mathrm{C} & 2.6020000 & -2.6580000 & 3.1250000 \\ \mathrm{C} & 1.4350000 & -2.8570000 & 3.9360000 \\ \mathrm{C} & 0.7630000 & -4.1420000 & 3.9090000\end{array}$




\begin{tabular}{|c|c|c|c|}
\hline $\mathrm{C}$ & 3.9020000 & -1.9620000 & \\
\hline $\mathrm{C}$ & 3.8850000 & -3.3220000 & 1.1520000 \\
\hline & 3.2030000 & -3.6910000 & 2.3310000 \\
\hline $\mathrm{C}$ & 2.5840000 & & 2.3650000 \\
\hline $\mathrm{C}$ & 1.3560000 & -5.1500000 & 3.0970000 \\
\hline $\mathrm{C}$ & 3.8650000 & -4.1580000 & 0.0000000 \\
\hline $\mathrm{C}$ & 3.1650000 & -5.3840000 & 0.0000000 \\
\hline $\mathrm{C}$ & 2.5590000 & & 1.2090000 \\
\hline $\mathrm{C}$ & -3.2480000 & -4.7620000 & 1.4290000 \\
\hline $\mathrm{C}$ & -2.6180000 & -4.3880000 & 2.6600000 \\
\hline $\mathrm{C}$ & 1.4290000 & -0.6520000 & 3.1040000 \\
\hline $\mathrm{C}$ & -1.4340000 & -5.0330000 & 3.1250000 \\
\hline $\mathrm{C}$ & -2.6220000 & -3.0490000 & \\
\hline $\mathrm{C}$ & 0.6810000 & -1.5810000 & 3.9360000 \\
\hline $\mathrm{C}$ & -0.6850000 & -4.1040000 & 3.9310000 \\
\hline $\mathrm{C}$ & -1.4390000 & -2.8310000 & 3.9440000 \\
\hline $\mathrm{C}$ & -0.7730000 & -1.5400000 & 3.9350000 \\
\hline $\mathrm{C}$ & 2.6640000 & 0.1150000 & 0.7090000 \\
\hline $\mathrm{C}$ & -2.6770000 & -5.8280000 & 0.7060000 \\
\hline $\mathrm{C}$ & -3.9160000 & -3.7560000 & 0.7080000 \\
\hline $\mathrm{C}$ & 1.5210000 & 0.8280000 & 1.1730000 \\
\hline $\mathrm{C}$ & -1.4770000 & -6.4690000 & 1.1520000 \\
\hline $\mathrm{C}$ & -3.8950000 & -2.3950000 & 1.1540000 \\
\hline $\mathrm{C}$ & 0.8420000 & 0.4040000 & 2.3160000 \\
\hline $\mathrm{C}$ & -0.8220000 & -6.0560000 & 2.3320000 \\
\hline $\mathrm{C}$ & -3.2180000 & -2.0250000 & 2.3310000 \\
\hline $\mathrm{C}$ & -0.5990000 & 0.4560000 & 2.3360000 \\
\hline $\mathrm{C}$ & 0.5860000 & -6.1250000 & 2.3640000 \\
\hline $\mathrm{C}$ & -2.5750000 & -0.7650000 & 2.3500000 \\
\hline $\mathrm{C}$ & -1.3510000 & -0.5530000 & 3.0820000 \\
\hline $\mathrm{C}$ & 0.8900000 & 1.4520000 & 0.0000000 \\
\hline $\mathrm{C}$ & -0.7360000 & -6.8560000 & 0.0000000 \\
\hline & -3.8790000 & -1.5540000 & 0.0000000 \\
\hline
\end{tabular}




\begin{tabular}{|c|c|c|c|}
\hline $\mathrm{C}$ & -0.8240000 & 1.4330000 & \\
\hline $\mathrm{C}$ & 0.6750000 & -6.8390000 & 0.0000000 \\
\hline & -3.1770000 & -0.3390000 & 0.0000000 \\
\hline $\mathrm{C}$ & -1.3510000 & & .2180000 \\
\hline $\mathrm{C}$ & 1.3260000 & -6.5100000 & 1.2090000 \\
\hline $\mathrm{C}$ & -2.5550000 & 0.0630000 & 1.2050000 \\
\hline $\mathrm{C}$ & 0.0210000 & 2.6770000 & 0.0000000 \\
\hline $\mathrm{C}$ & 0.0200000 & 3.5460000 & 1.2530000 \\
\hline $\mathrm{C}$ & 1.2740000 & 4.4420000 & 1.2440000 \\
\hline $\mathrm{C}$ & 1.2680000 & 5.3370000 & 0.0000000 \\
\hline $\mathrm{C}$ & -1.2540000 & 5.3190000 & 0.0000000 \\
\hline $\mathrm{C}$ & -1.2460000 & 4.4250000 & 1.2460000 \\
\hline $\mathrm{C}$ & 0.0010000 & 6.2150000 & 0.0000000 \\
\hline $\mathrm{H}$ & 0.0230000 & 2.9320000 & 2.1410000 \\
\hline $\mathrm{H}$ & 1.2780000 & 5.0540000 & 2.1410000 \\
\hline $\mathrm{H}$ & 2.1690000 & 3.8270000 & 1.2600000 \\
\hline $\mathrm{H}$ & 2.1500000 & 5.9680000 & 0.0000000 \\
\hline $\mathrm{H}$ & -2.1450000 & & 0.0000000 \\
\hline $\mathrm{H}$ & -2.1320000 & 3.7980000 & 1.2650000 \\
\hline $\mathrm{H}$ & -1.2580000 & 5.0380000 & 2.1410000 \\
\hline $\mathrm{H}$ & -0.0040000 & 6.8560000 & 0.8690000 \\
\hline $\mathrm{C}$ & 3.2270000 & -0.9530000 & -1.4190000 \\
\hline $\mathrm{C}$ & 2.5980000 & & \\
\hline $\mathrm{C}$ & 2.6020000 & -2.6580000 & -3.1250000 \\
\hline $\mathrm{C}$ & 1.4350000 & -2.8570000 & -3.9360000 \\
\hline $\mathrm{C}$ & 0.7630000 & -4.1420000 & -3.9090000 \\
\hline $\mathrm{C}$ & 3.9020000 & -1.9620000 & -0.7020000 \\
\hline $\mathrm{C}$ & 3.8850000 & -3.3220000 & -1.1520000 \\
\hline $\mathrm{C}$ & 3.2030000 & -3.6910000 & -2.3310000 \\
\hline $\mathrm{C}$ & 2.5840000 & -4.9560000 & -2.3650000 \\
\hline $\mathrm{C}$ & 1.3560000 & -5.1500000 & -3.0970000 \\
\hline $\mathrm{C}$ & 2.5590000 & -5.7890000 & -1.2090000 \\
\hline 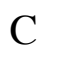 & -3.2480000 & -4.7620000 & -1.4290000 \\
\hline
\end{tabular}




\begin{tabular}{|c|c|c|c|}
\hline & -2.6180000 & -4.3880000 & -2.6600000 \\
\hline & 1.4290000 & -0.6520000 & -3.1040000 \\
\hline & -1.4340000 & -5.0330000 & -3.1250000 \\
\hline C & -2.6220000 & -3.0490000 & -3.1310000 \\
\hline $\mathrm{C}$ & 0.6810000 & -1.5810000 & -3.9360000 \\
\hline $\mathrm{C}$ & -0.6850000 & -4.1040000 & -3.9310000 \\
\hline $\mathrm{C}$ & -1.4390000 & -2.8310000 & -3.9440000 \\
\hline $\mathrm{C}$ & -0.7730000 & -1.5400000 & -3.9350000 \\
\hline $\mathrm{C}$ & 2.6640000 & 0.1150000 & -0.7090000 \\
\hline $\mathrm{C}$ & -2.6770000 & -5.8280000 & -0.7060000 \\
\hline $\mathrm{C}$ & -3.9160000 & -3.7560000 & -0.7080000 \\
\hline C & 1.5210000 & 0.8280000 & -1.1730000 \\
\hline $\mathrm{C}$ & -1.4770000 & -6.4690000 & -1.1520000 \\
\hline C & -3.8950000 & -2.3950000 & -1.1540000 \\
\hline $\mathrm{C}$ & 0.8420000 & 0.4040000 & -2.3160000 \\
\hline $\mathrm{C}$ & -0.8220000 & -6.0560000 & -2.3320000 \\
\hline C & -3.2180000 & -2.0250000 & -2.3310000 \\
\hline $\mathrm{C}$ & -0.5990000 & 0.4560000 & -2.3360000 \\
\hline C & 0.5860000 & -6.1250000 & -2.3640000 \\
\hline $\mathrm{C}$ & -2.5750000 & -0.7650000 & -2.3500000 \\
\hline $\mathrm{C}$ & -1.3510000 & -0.5530000 & -3.0820000 \\
\hline $\mathrm{C}$ & -1.3510000 & 0.8290000 & -1.2180000 \\
\hline $\mathrm{C}$ & 1.3260000 & -6.5100000 & -1.2090000 \\
\hline $\mathrm{C}$ & -2.5550000 & 0.0630000 & -1.2050000 \\
\hline $\mathrm{C}$ & 0.0200000 & 3.5460000 & -1.2530000 \\
\hline C & 1.2740000 & 4.4420000 & -1.2440000 \\
\hline $\mathrm{C}$ & -1.2460000 & 4.4250000 & -1.2460000 \\
\hline $\mathrm{H}$ & 0.0230000 & 2.9320000 & -2.1410000 \\
\hline $\mathrm{H}$ & 1.2780000 & 5.0540000 & -2.1410000 \\
\hline $\mathrm{H}$ & 2.1690000 & 3.8270000 & -1.2600000 \\
\hline $\mathrm{H}$ & -2.1320000 & 3.7980000 & -1.2650000 \\
\hline $\mathrm{H}$ & -1.2580000 & 5.0380000 & -2.1410000 \\
\hline $\mathrm{H}$ & -0.0040000 & 6.8560000 & -0.8690000 \\
\hline
\end{tabular}

